\title{
INTERNATIONAL CENTRE FOR THEORETICAL PHYSICS
}

\author{
CUMULANT APPROACH \\ TO DYNAMICAL CORRELATION FUNCTIONS \\ AT FINITE TEMPERATURES
}

Tran Minh-Tien

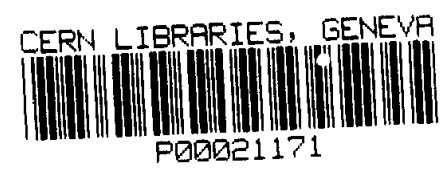

INTERNATIONAL ATOMIC ENERGY AGENCY

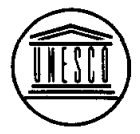

UNITED NATIONS EDUCATIONAL, SCIENTIFIC AND CULTURAL ORGANIZATION 


$\mathrm{IC} / 93 / 389$

\author{
International Atomic Energy Agensy \\ and \\ United Nations Educational Scientific and Cultural Organization \\ INTERNATIONAL CENTRE FOR THEORETICAL PHYSICS
}

\title{
CUMULANT APPROACH \\ TO DYNAMICAL CORRELATION FUNCTIONS \\ AT FINITE TEMPERATURES
}

\author{
Tran Minh-Tien ${ }^{1}$ \\ International Centre for Theoretical Physics, Trieste, Italy.
}

\begin{abstract}
A new theoretical approach, based on the introduction of cumulants, to calculate thermodynamic averages and dynamical correlation functions at finite temperatures is developed. The method is formulated in Liouville instead of Hilbert space and can be applied to operators which do not require to satisfy fermion or boson commutation relations. The application of the partitioning and projection methods for the dynamical correlation functions is discussed. The present method can be applied to weakly as well as to strongly correlated systems.
\end{abstract}

MIRAMARE - TRIESTE

November 1993

\footnotetext{
${ }^{1}$ Address after 30 November 1993: Max-Planck-Institut für Physik Komplexer Systeme, Heisenbergstrasse 1, D-70569 Stuttgart, Germany.
} 


\section{Introduction}

The discovery of high $T_{c}$ superconductors (HTSC) has greatly increased the theoretical interest in the physics of strongly correlated systems. However, the theoretical treatment of strongly correlated systems has turned out to be extremely difficult due to the Wick's theorem cannot apply at least in the standard form. Recently, a new theoretical approach $[1,2]$ (see also the review in [3]) based on the introduction of cumulants was developed which can be applied to weakly and strongly correlated systems. However, the approach still is restricted only to zero temperature.

In this paper we develop the approach at finite temperatures. Using the contour integral formalism [4] the formulation of thermodynamic averages and dynamical correlation functions at finite temperatures is given in terms of cumulants. The introduction of cumulants ensures that any approximations for dynamical correlation functions and thermodynamic averages in this method are "size consistent". The method is not restricted to operators which obey fermion or boson commutation relations and is suitable for applying the partitioning and projection methods.

The paper is organized as follows. In Sec. 2 we formulate thermodynamic averages and dynamical correlation functions at finite temperatures in terms of cumulants. The application of the partitioning and projection methods for the correlation functions is given in Sec.3. Finally, in Sec.4 the summary and concluding remarks are presented.

\section{Dynamical correlation functions}

Starting point is a Hamiltonian $H$ which describes a many-particle system, which is split according to

$$
H=H_{0}+H_{1},
$$

which $H_{1}$ being comparatively small. It should be reemphasized that no specify assumptions with respect to the above split of $H$ is required.

Consider the functional

$$
f[h]=\ln <T_{\tau}\left\{\mathrm{e}^{-\int_{0}^{\beta} d \tau\left(H-\sum_{\nu} h_{\nu}(\tau) A_{\nu}\right)}\right\} \mathrm{e}^{\beta H_{0}}>_{0} .
$$

Here $\left\langle\cdots>_{0}\right.$ indicates a thermodynamic average with respect to $H_{0}$, $\therefore$.e.

$$
<\cdots>_{0}=\frac{\operatorname{Tr}\left\{\cdots \mathrm{e}^{-\beta H_{0}}\right\}}{\operatorname{Tr}\left\{\mathrm{e}^{-\beta H_{0}}\right\}},
$$

$T_{\tau}$ is the time ordering operator for imaginary times $\tau$. The functional $f[h]$ has the same structure as the one using by Becker and Brening [2] in the formulation of dynamical correlation functions in terms of cumulants in zero temperature.

The quantity $f[h]$ is a functional of the fields $h_{\nu}(\tau)$. Its first functional derivatives with respect to the external fields $h_{\nu}(\tau)$ can be written in the forms

$$
\left(\frac{\delta f}{\delta h_{\nu}(\lambda)}\right)_{h=0}=\frac{<\mathrm{e}^{-\beta H} A_{\nu}(\lambda) \mathrm{e}^{\beta H_{0}}>_{0}}{<\mathrm{e}^{-\beta H} \mathrm{e}^{\beta H_{0}}>_{0}}=<A_{\nu}(\lambda)>, \quad(0 \leq \lambda \leq \beta),
$$




$$
\begin{aligned}
\left(\frac{\delta^{2} f}{\delta h_{\nu}(\lambda) \delta h_{\mu}\left(\lambda^{\prime}\right)}\right)_{h=0}= & \frac{<\mathrm{e}^{-\beta H} A_{\nu}(\lambda) A_{\mu}\left(\lambda^{\prime}\right) \mathrm{e}^{\beta H_{0}}>_{0}}{<\mathrm{e}^{-\beta H} \mathrm{e}^{\beta H_{0}}>_{0}}- \\
& -\frac{<\mathrm{e}^{-\beta H} A_{\nu}(\lambda) \mathrm{e}^{\beta H_{0}}>_{0}}{<\mathrm{e}^{-\beta H} \mathrm{e}^{\beta H_{0}}>_{0}} \frac{<A_{\mu}(\lambda) \mathrm{e}^{\beta H_{0}}>_{0}}{<H} \mathrm{e}^{\beta H_{0}}>_{0} \\
= & <A_{\nu}(\lambda) A_{\mu}\left(\lambda^{\prime}\right)>-<A_{\nu}(\lambda)><A_{\mu}\left(\lambda^{\prime}\right)>= \\
= & <\delta A_{\nu}(\lambda) \delta A_{\mu}\left(\lambda^{\prime}\right)>, \quad\left(0 \leq \lambda^{\prime} \leq \lambda \leq \beta\right) .
\end{aligned}
$$

Here $\langle\cdots\rangle$ denotes a thermodynamic average with respect to $H$, and $\delta A_{\nu}(\lambda)=$ $A_{\nu}(\lambda)-\left\langle A_{\nu}(\lambda)\right\rangle$, where $A_{\nu}(\lambda)=\exp (\lambda H) A_{\nu} \exp (-\lambda H)$. Note that the thermodynamic averages $\left\langle A_{\nu}\right\rangle$ and $\left\langle\delta A_{\nu} \delta A_{\mu}\right\rangle$ can be calculated by the expressions

$$
\begin{aligned}
\left\langle A_{\nu}\right\rangle & =\frac{1}{\beta} \int_{0}^{\beta} d \lambda\left\langle A_{\nu}(\lambda)\right\rangle \\
\left.<\delta A_{\nu} \delta A_{\mu}\right\rangle & =\frac{1}{\beta} \int_{0}^{\beta} d \lambda<\delta A_{\nu}(\lambda) \delta A_{\mu}(\lambda)>
\end{aligned}
$$

On the other hand, Ec. (2) can be rewritten as

$$
f[h]=\ln <T_{\tau}\left\{\mathrm{e}^{-\int_{0}^{\beta} d \tau\left(\mathcal{L}_{0}+H_{1}-\sum_{\nu} h_{\nu}(\tau) A_{\nu}\right)}\right\}>_{0},
$$

where $\mathcal{L}_{0}$ is the Liouville operator for $H_{0}$, i.e. $\mathcal{L}_{0} A=\left[H_{0}, A\right]$.

For the following we express $f[h]$ in terms of cumulants. The cumulant expression for $f[h]$ is (see also $[1,5]$ )

$$
f[h]=<T_{\tau}\left\{\mathrm{e}^{-\int_{0}^{\beta} d \tau\left(\mathcal{L}_{0}+H_{1}-\sum_{\nu} h_{\nu}(r) A_{\nu}\right)}\right\}-1>_{0}^{c},
$$

where the index $c$ denotes the cumulant average.

From Eqs.(3),(4),(8) we get

$$
\begin{array}{r}
<A_{\nu}(\lambda)>=<\mathrm{e}^{-(\beta-\lambda)\left(\mathcal{L}_{0}+H_{1}\right)} A_{\nu} \mathrm{e}^{-\lambda\left(\mathcal{L}_{0}+H_{1}\right)}>_{0}^{c}, \quad(0 \leq \lambda \leq \beta) \\
<\delta A_{\nu}(\lambda) \delta A_{\mu}\left(\lambda^{\prime}\right)>=<\mathrm{e}^{-(\beta-\lambda)\left(\mathcal{L}_{0}+H_{1}\right)} A_{\nu} \mathrm{e}^{-\left(\lambda-\lambda^{\prime}\right)\left(\mathcal{L}_{0}+H_{1}\right)} A_{\mu} \mathrm{e}^{-\lambda^{\prime}\left(\mathcal{L}_{0}+H_{1}\right)}>_{0}^{c} \\
\left(0 \leq \lambda^{\prime} \leq \lambda \leq \beta\right)
\end{array}
$$

Using the contour integral representation [4] and Eq.(9), Eq.(5) can be written as

$$
\begin{aligned}
& <A_{\nu}>=\frac{1}{\beta} \int_{0}^{\beta} d \lambda<\mathrm{e}^{-(\beta-\lambda)\left(\mathcal{L}_{0}+H_{1}\right)} A_{\nu} \mathrm{e}^{-\lambda\left(\mathcal{L}_{0}+H_{1}\right)}>_{0}^{c}= \\
& =\frac{1}{\beta} \int_{0}^{\beta} d \lambda \oint \frac{d z_{1}}{2 \pi i} \mathrm{e}^{-(\beta-\lambda) z_{1}} \oint \frac{d z_{2}}{2 \pi i} \mathrm{e}^{-\lambda z_{2}}<\frac{1}{z_{1}-\left(\mathcal{L}_{0}+H_{1}\right)} A_{\nu} \frac{1}{z_{2}-\left(\mathcal{L}_{0}+H_{1}\right)}>_{0}^{c} .
\end{aligned}
$$

Here the contour integrals surround all (discrete or continuous) singularities of $\left(z_{1}-\left(\mathcal{L}_{0}+H_{1}\right)\right)^{-1}$ and $\left(z_{2}-\left(\mathcal{L}_{0}+H_{1}\right)\right)^{-1}$ respectively. 
Performing the integration on $\lambda$ in Eq.(11) we get

$$
<A_{\nu}>=\oint \oint \frac{d z_{1} d z_{2}}{(2 \pi i)^{2}} \frac{1}{\beta} \frac{\mathrm{e}^{-\beta z_{2}}-\mathrm{e}^{-\beta z_{1}}}{z_{1}-z_{2}}<\frac{1}{z_{1}-\left(\mathcal{L}_{0}+H_{1}\right)} A_{\nu} \frac{1}{z_{2}-\left(\mathcal{L}_{0}+H_{1}\right)}>_{0}^{c} .
$$

Since the contours surround only the singularities of $\left(z_{1}-\left(\mathcal{L}_{0}+H_{1}\right)\right)^{-1}$ or $\left(z_{2}-\left(\mathcal{L}_{0}+H_{1}\right)\right)^{-1}$, we can perform one of the contour integrals by residues with result

$$
<A_{\nu}>=\oint \frac{d z}{\pi i} \frac{\mathrm{e}^{-\beta z}}{\beta}<\frac{1}{z-\left(\mathcal{L}_{0}+H_{1}\right)} A_{\nu} \frac{1}{z-\left(\mathcal{L}_{0}+H_{1}\right)}>_{0}^{c}
$$

Using the power series expansion

$$
\begin{aligned}
\frac{1}{z-\left(\mathcal{L}_{0}+H_{1}\right)} & =\frac{1}{z} \sum_{n=0}^{\infty} \frac{\left(\mathcal{L}_{0}+H_{1}\right)^{n}}{z^{n}}=\frac{1}{z}+\frac{1}{z}\left(\mathcal{L}_{0}+H_{1}\right) \frac{1}{z-\left(\mathcal{L}_{0}+H_{1}\right)} \\
& =\frac{1}{z}+\frac{1}{z} \frac{1}{z-\left(\mathcal{L}_{0}+H_{1}\right)}\left(\mathcal{L}_{0}+H_{1}\right)
\end{aligned}
$$

and $<\mathcal{L}_{0} \cdots>_{0}=<\cdots \mathcal{L}_{0}>_{0}=0$, Eq.(13) can be rewritten in the form

$$
<A_{\nu}>=\oint \frac{d z}{\pi i} \frac{\mathrm{e}^{-\beta z}}{\beta z^{2}}<\Omega^{\dagger}(z) A_{\nu} \Omega(z)>_{0}^{c}
$$

where

$$
\Omega(z)=1+\frac{1}{z-\left(\mathcal{L}_{0}+H_{1}\right)} H_{1}
$$

By the similar procedure, from Eqs.(6),(10) we get

$$
<\delta A_{\nu} \delta A_{\mu}>=\oint \frac{d z}{\pi i} \frac{e^{-\beta z}}{\beta z^{2}}<\Omega^{\dagger}(z) A_{\nu} A_{\mu} \Omega(z)>_{0}^{c}
$$

Note that Eq.(15) is valid for arbitrary operator $A_{\nu}, A_{\mu}$. Replacing in Eq.(15) $\delta A_{\mu}$ by $\delta A_{\mu}(-t)$ we get

$$
<\delta A_{\nu} \delta A_{\mu}(-t)>=\oint \frac{d z}{\pi i} \frac{\mathrm{e}^{-\beta z}}{\beta z^{2}}<\Omega^{\dagger}(z) A_{\nu} A_{\mu}(-t) \Omega(z)>_{0}^{c}
$$

On the other hand, $<\delta A_{\nu} \delta A_{\mu}(-t)>=<\delta A_{\nu}(t) \delta A_{\mu}>\equiv G_{\nu \mu}(t)$ is the dynamical correlation function. Using

$$
A_{\mu}(-t)=\mathrm{e}^{-i H t} A_{\mu} \mathrm{e}^{i H t}=\mathrm{e}^{-i \mathcal{L} t} A_{\mu}
$$

we get the cumulant expression for the correlation function $G_{\nu \mu}(t)$

$$
G_{\mu \nu}(t)=\oint \frac{d z}{\pi i} \frac{\mathrm{e}^{-\beta z}}{\beta z^{2}}<\Omega^{\dagger}(z) A_{\nu}\left(\mathrm{e}^{-i \mathcal{L} t} A_{\mu}\right)^{\bullet} \Omega(z)>_{0}^{c}
$$

Here $\mathcal{L}$ is the Liouville operator corresponding to $H$ and the symbol $(\cdots)^{\bullet}$ indicates the way in which cumulants have to be taken in Eq.(17), i.e., each term in the power series expansion of the expression in the parentheses with black dot has to be treated as a single operator entity subjected to the cumulant calculation. 
In zero temperature limit, $\beta \rightarrow \infty$, only the pole $z=0$ gives nonzero residue in the integrals in Eqs.(14) and (17). Thus at zero temperature we obtain

$$
\begin{aligned}
<A_{\nu}> & =<\Omega^{\dagger} A_{\nu} \Omega>_{0}^{c} \\
G_{\nu \mu}(t) & =<\Omega^{\dagger} A_{\nu}\left(\mathrm{e}^{-i \mathcal{L} t} A_{\mu}\right)^{\bullet} \Omega>_{0}^{c} .
\end{aligned}
$$

Here $\langle\cdots\rangle_{0}$ becomes the ground state average and

$$
\Omega=1+\lim _{z \rightarrow 0} \frac{1}{z-\left(\mathcal{L}_{0}+H_{1}\right)} H_{1} .
$$

The expressions (18) and (19) are exactly the ones which have been previously derived by Becker and Brening [2] in zero temperature. As discussed in [3] $\Omega(z)$ can be considered as the generalized wave vector. At zero temperature it transforms the ground state of the unperturbed system into that of the interacting system. However, at finite temperatures $\Omega(z)$ does not obey this property. Roughly speaking, it transform the set of eigenstates of the unperturbed system into a new set of states, which are not eigenstates of the interacting system. However, the combination of thermodynamic averages of arbitrary operator in the new set with weight $\exp (-\beta z) / \beta z^{2}$ gives exactly its thermodynamic average in the interacting system. It should be reemphasized that the introduction of cumulants ensures that statistically independent processes are eliminated from the calculations of thermodynamic averages or of dynamical correlation functions. The expressions (18) and (19) may serve as an alternative approach for the calculations of thermodynamic averages and dynamical correlation functions as compared with the standard diagrammatic technique. They are very useful particularly for systems where the Wick's theorem is not valid.

\section{Application of partitioning and projection meth- ods}

Expression (17) is a reformulation of the correlation function in terms of cumulants at finite temperatures. It is very useful and applicable even for systems where the Wick's theorem is not valid. For arbitrary correlation function $G_{A B}(t)=\langle\delta A(t) \delta B\rangle$, its Fourier transform is given by

$$
G_{A B}=\oint \frac{d z}{\pi i} \frac{\mathrm{e}^{-\beta z}}{\beta z^{2}}<\Omega^{\dagger}(z) A\left(\frac{1}{\omega-\mathcal{L}} B\right)^{\bullet} \Omega(z)>_{0}^{c} .
$$

Eq.(20) can be applied to arbitrary operators $A, B$, i.e. neither of both has to satisfy fermion or boson commutation relations.

Let us introduce the following bilinear form in Liouville space

$$
\left(A^{\dagger} \mid B\right)=\oint \frac{d z}{\pi i} \frac{\mathrm{e}^{-\beta z}}{\beta z^{2}}<\Omega^{\dagger}(z) A(B)^{\bullet} \Omega(z)>_{0}^{c},
$$

where $A, B$ are operators, i.e., elements of the Liouville space. Note that $\left(A^{\dagger} \mid A\right)=$ $<A^{2}>-<A>^{2} \geq 0$, thus Eq.(21) also defines a scalar product in the Liouville space. In terms of the scalar product $(21)$, the correlation function $G_{A B}(\omega)$ changes into

$$
G_{A B}(\omega)=\left(A^{\dagger} \mid \frac{1}{\omega-\mathcal{L}} B\right)
$$


Eq.(22) is in a proper form to be evaluated by the Mori-Zwanzig projection technique $[6,7]$ based on the partitioning concept [8] (see also [3]). In the framework of that formalism the dynamic of the correlation function $G_{A B}(\omega)$ is restricted to a relevant subspace of Liouville space which is spanned by the operators $\left\{A_{\nu}\right\}$. Within this subspace $G_{A B}(\omega)$ can be evaluated exactly. For the calculation we introduce the projection operator $\mathcal{P}$

$$
\left.\mathcal{P}=\sum_{\mu \nu} \mid A_{\mu}\right) \chi_{\mu \nu}^{-1}\left(A_{\nu} \mid\right.
$$

where $\chi_{\mu \nu}=\left(A_{\mu} \mid A_{\nu}\right)$ is the so-called susceptibility matrix.

In the projection technique the dynamical correlation function (22) is approximated by

$$
G_{A B}(\omega)=\left(A \mid \mathcal{P} \frac{1}{\omega-\mathcal{P} \mathcal{L P}} \mathcal{P} B\right) .
$$

In this approximation $G_{A B}(\omega)$ can be exactly expressed in the following form [9]

$$
G_{A B}(\omega)=c_{A}^{\dagger}[\omega \hat{\chi}-\hat{F}]^{-1} c_{B},
$$

where $F_{\mu \nu}=\left(A_{\mu} \mid \mathcal{L} A_{\nu}\right)$ is the so-called frequency matrix and $c_{A}^{\mu}=\left(A_{\mu} \mid A\right), c_{B}^{\mu}=\left(A_{\mu} \mid B\right)$ are so-called coupling vectors.

In this way the calculation of the correlation function has been reduced to calculations of the coupling vectors, susceptibility and frequency matrices, which are evaluated in terms of cumulants. By successively enlarging the dimension of the relevant subspace spanned by $\left\{A_{\mu}\right\}$ we can improve the approximation of correlation functions. The different between the above method based on the introduction of cumulants and the standard Mori-Zwanzig projection method is discussed elsewhere [3].

\section{Conclusion}

In summary, we have given the formulation of thermodynamic averages and dyuamical correlation functions at finite temperatures in terms of cumulants based on the contour integral formalism. The formulation can be applied to operators which do not require to satisfy fermion or boson commutation relations, thus it is applicable for weakly as well as for strongly correlated systems. At zero temperature the expressions for thermodynamic averages and dynamical correlation functions have been reduced exactly to the ones have been previously derived by Becker and Brening [2]. The application of the partitioning and projection methods for the correlation functions is considered. In the framework of that formalism the dynamical correlation function is exactly calculated within the relevant subspace of Liouville space. It expresses in the form containing only the coupling vectors, susceptibility and frequency matrices, which are evaluated in terms of cumulants. Some applications of the method at zero temperature have already been given (see [3]). However, here we do not present applications of the method at finite temperatures. They will be given elsewhere.

\section{Acknowledgments}

The aithor should like to thank Professor Yu Lu for encouragement in the research and the warm hospitality. The author would also like to acknowledge Professor Abdus Salam and the International Atomic Energy Agency and UNESCO for the hospitality at the International Centre for Theoretical Physics (Trieste, Italy). 


\section{References}

[1] Becker K.W., and Fulde P., Z.Phys.B 72, 423 (1988).

[2] Becker K.W., and Brening W., Z.Phys.B 79, 195 (1990).

[3] Fulde P.: Electron Correlation in Molecules and Solids, Springer Ser., Solid-state Sci., vol.100 (Springer-Verlag 1991).

[4] Keiter H., and Morandi G., Phys.Rep. 109, 227 (1984).

[5] Kubo R., J.Phys.Soc.Jpn. 17, 1100 (1962).

[6] Mori H., Prog.Theor.Phys. (Kyoto) 34, 423 (1965).

[7] Zwanzig R.: in Lectures in Theoretical Physics, Interscience, New York 1961, vol.3.

[8] Löwdin P.O.: in Supercomputer Simulations in Chemistry, ed. by M.Dupuis, Lect. Notes Chem. Phys. vol.44, Springer-Verlag 1986.

[9] Forster F.: Hydrodynamic Fluctuations, Broken Symmetry and Correlation Functions, Benjamin, New York 1978. 